\title{
CUANTIFICACIÓN DE TIOLES LIBRES Y SUPEROXIDO DISMUTASA (SOD) EN EXTRACTOS METANOLICOS DE PLANTAS DE LAS FAMILIAS ASTERACEAE, EUPHORBIACEAE Y PIPERACEAE
}

\section{QUANTIFICATION OF FREE THIOLS AND SUPEROXIDE DISMUTASE (SOD) IN METHANOLIC PLANT EXTRACTS FROM THE FAMILIES ASTERACEAE, EUPHORBIACEAE AND PIPERACEAE}

\author{
Yeimy Lizeth BETANCUR', Oscar M. MOSQUERA'* \\ ${ }^{1}$ Grupo de Biotecnología-Productos Naturales (GBPN) Escuela de Tecnología Química \\ Universidad Tecnológica de Pereira (UTP). Carrera 27 No 10-02. Barrió Los Álamos. Pereira- \\ Colombia.
}

Historia del Artículo

Recibido: Julio 26, 2016

Evaluado: Diciembre 29, 2016

Aceptado: Enero 24, 2017

Disponible: Febrero 09, 2017

*Autor Corresponsal. E-mail: omosquer@utp.edu.co

Resumen |

Dada la toxicidad de las especies reactivas de oxígeno (ROS) y la actual importancia de los fitocompuestos, en este trabajo se evaluaron 32 extractos metanólicos obtenidos por maceración pasiva de la parte área de especies pertenecientes a las familias Asteraceae, Euphorbiaceae y Piperaceae. A estos extractos se les evaluó la concentración de tioles libres de bajo peso molecular, mediante el método de DTNB, usando glutationa (GSH) como tiol de referencia. A los que presentaron mayor concentración se les evaluó la capacidad de disminuir el radical superóxido a través de la inhibición de la superoxido dismutasa (SOD) y seguidamente se determinó la capacidad antioxidante por el método del radical DPPH'. Las especies de las familias Asteraceae y Piperaceae se destacaron por su alto contenido de compuestos de naturaleza tiol. Además, estas especies también se destacaron en el ensayo de actividad antioxidante frente al radical $\mathrm{DPPH}^{\circ}$ obteniendo valores para el porcentaje de actividad antioxidante similares a la hidroquinona que fue usada como control positivo.

Palabras Clave: Biodiversidad, bioprospección, Radicales libres, antioxidantes , tioles, fitocompuestos.

\section{Abstract}

Given the toxicity of the reactive oxygen species (ROS) and the current importance of the phytocompounds, in this work were evaluated 32 methanolic extracts obtained by passive maceration of the part area of the species belonging to the family Asteraceae, Euphorbiaceae and Piperaceae. These extracts were evaluated free of low thiol concentration, molecular weight DTNB method using glutathione (GSH) as tiol reference. The ability to reduce superoxide radical through inhibition of superoxide dismutase (SOD) was evaluated to those presenting higher tiol contents and the antioxidant capacity by the DPPH radical method was then determined. Species of the families Asteraceae and Piperaceae stood out for its high content of thiol-related compounds. In addition, these families were also highlighted in the antioxidant activity assay against the radical DPPH' obtaining antioxidant activity percentage values similar to hydroquinone which was used as a positive control.

Keywords: Biodiversity, bioprospecting, radical free, antioxidant, thiols, phytocompounds.

\section{INTRODUCCIÓN |}

Las especies reactivas de oxígeno (ROS) representan un grupo de moléculas químicamente derivadas del oxígeno (Nordberg \& Arnér, 2001) los cuales son generados normalmente durante los procesos metabólicos de los seres vivos. Estas especies están compuestas por un grupo de moléculas denominadas radicales libres, denominados como átomos o grupos de átomos con un número impar de electrones, los cuales presentan una alta reactividad química y pueden generar una cadena de reacciones al entrar en contacto con otras moléculas, cuando se presenta una generación excesiva de ROS y/o insuficiencia en el sistema de defensa antioxidante, se produce un fenómeno denominado estrés oxidativo, al cual se le atribuye su participación en la generación de diversas enfermedades (Wu et al, 2013). 
En la búsqueda por mitigar los efectos causados por la acción de los radicales libres sobre las biomoléculas, se han implementado antioxidantes de origen sintético como el butil-hidroxitolueno y el butil-hidroxianisol, los cuales pueden ser acumulados en el organismo causando graves daños, entre los cuales se les atribuye la generación de carcinogénesis (Gao et al, 2012), Por este motivo las actuales investigaciones apuntan a la búsqueda de fitocompuestos que presenten dicha actividad, siendo las plantas la principal fuente de estudio debido a la gran variadad de metabolitos secundarios que estas producen entre los cuales se destacan polifenoles, flavonoides, cumarinas, antronas y antraquinonas, principalmente (Yen, 2000).

Adicionalmente, se ha evidenciado que los compuestos tipo tiol son de gran importancia en el equilibrio redox de las células. Estos corresponden a uno de los grupos nucleofílicos más fuertes en las células, los cuales participan en reacciones químicas, a tal punto que, las moléculas que contienen grupos tiol dentro de su estructura, desempeñan un rol primario en la homeostasis redox celular, en el control de las actividades enzimáticas y en la destoxificación de especies reactivas de oxígeno, nitrógeno y xenobióticos (Masi et al, 2008).

Teniendo en cuenta la biodiversidad de la Eco Región cafeterea en el presente estudio se utilizaron 32 especies vegetales recolectadas en dos reservas naturales de la región. Las especies en estudio se les determino la concentración de tioles libres presentes en sus extractos metanólicos, teniendo en cuenta estas concentraciones se eligieron las de mayor contenido de tioles y se evaluó su capacidad antioxidante teniendo en cuenta la capacidad de estos para dismutar el radical superóxido y su actividad frente al radical $\mathrm{DPPH}^{*}$ con el objeto de establecer una relación entre el contenido de estas moléculas y su capacidad antioxidante.

\section{MATERIALES Y MÉTODOS |}

\section{Obtención del material vegetal}

Se trabajó con la parte aérea de las 32 especies mencionadas en la tabla 1, las cuales fueron recolectadas en las reservas naturales de la ecorregión cafetera: Parque Natural Regional Ucumarí $4^{\circ} 43^{\prime} 22.0^{\prime \prime} \mathrm{N}, 75^{\circ} 35^{\prime} 42.9^{\prime \prime} \mathrm{W}$ y la Reserva Natural Bremen-La Popa $4^{\circ} 40^{\prime} 48.6^{\prime \prime} \mathrm{N}$ y $75^{\circ} 37^{\prime}$ 32.7"W; la identificación fue realizada por el taxónomo Francisco Javier Roldan y voucher fue depositado en el herbario de la Universidad de Antioquia.

Los extractos vegetales fueron obtenidos por maceración pasiva con solventes orgánicos (hexano, diclorometano y metanol) de polaridad creciente siguiendo el procedimiento descrito por Niño et al., 2006, el solvente fue evaporado en un rotaevaporador Laborota 4003 y finalmente los extractos secos fueron almacenados a $4^{\circ} \mathrm{C}$, para la realización de los ensayos de este estudio.

\section{Caracterización fitoquímica preliminar por cromatografía de capa delgada}

La evaluación fitoquímica de los extractos metanólicos crudos fue realizada por marcha fitoquímica a través de cromatografía de capa delgada (CCD) en placas de aluminio de sílica gel $60 \mathrm{~F}_{254}$ de acuerdo a la metodología descrita por Wagner y Bladt (1996). Cada extracto se sembró a una concentración de 10000 ppm y fueron desarrolladas con el eluente cloroformo-isopropanol (95:5). Los alcaloides, esteroles, terpenos, saponinas, fenoles y flavonoides se detectaron mediante las siguientes reacciones de caracterización: Dragendorff, anisaldehído-ácido sulfúrico, vainillina $1 \%$ en ácido sulfúrico-etanol, cloruro férrico al $1 \%$ y tricloruro de aluminio al $2 \%$, respectivamente, comparando estos resultados con los patrones correspondientes a cada núcleo fitoquímco. Todas las determinaciones se realizaron por duplicado.

Tabla 1. Especies vegetales pertenecientes a las familias Asteraceae,

Euphorbiaceae y Piperaceae originarias de la Eco Región Cafetera.

\begin{tabular}{|c|c|c|c|}
\hline FAMILIA & ESPECIE & FJR & UTP \\
\hline \multirow{12}{*}{ Asteraceae } & Mikania banisteriae & 3965 & 124 \\
\hline & Mikania lloensis & 3977 & 136 \\
\hline & Tilesia baccata & 3974 & 133 \\
\hline & Pentacalya urbanii & 3963 & 122 \\
\hline & Lepidaploa lehamannii & 3976 & 135 \\
\hline & Citroniella acuminata & 3968 & 127 \\
\hline & Clibadium pentaneuron & 3966 & 125 \\
\hline & Baccharis trinervis & 3972 & 131 \\
\hline & Mikania leiostachya & 3175 & 22 \\
\hline & Aspiliaq quinquenervis & 3750 & 81 \\
\hline & Montanoa sp & 3749 & 80 \\
\hline & Citronella acuminata & 4049 & 195 \\
\hline \multirow{12}{*}{ Euphorbiaceae } & Hyeronima sp & 3971 & 130 \\
\hline & Alchornea brittonii & 3982 & 140 \\
\hline & Alchornea coelophylla & 3969 & 128 \\
\hline & Acalypha diversifolia & 3967 & 126 \\
\hline & Croton magdalenensis & 3736 & 70 \\
\hline & Alchornea glandulosa & 3742 & 75 \\
\hline & Sapium stylare & 3160 & 7 \\
\hline & Hyeronima antioquiensis & 3905 & 85 \\
\hline & Acalypha paltyphylla & 3910 & 90 \\
\hline & Alchormea grandiflora & 3727 & 63 \\
\hline & Acalypha macrostachya & 3738 & 72 \\
\hline & Hyeronnima macrocarpa & 3196 & 42 \\
\hline \multirow{8}{*}{ Piperaceae } & Piper crassinervium & 4021 & 167 \\
\hline & Piper pesaresanum & 3996 & 148 \\
\hline & Piper umbellatum & 4012 & 163 \\
\hline & Peperomia acuminata & 4002 & 154 \\
\hline & Piper $s p$ & 4007 & 158 \\
\hline & Piper glanduligerum & 4026 & 172 \\
\hline & Piper calceolarium & 4048 & 194 \\
\hline & Piper Daniel-gonzalezii & 4051 & 197 \\
\hline
\end{tabular}

\section{Cuantificación de tioles libres}

Se usó el reactivo ácido 5,5'-Ditiobis-(2-nitobenzóico) (DTNB), conocido como el reactivo de Ellman's, compuesto que posee un enlace bisulfito, mediante el cual se lleva a cabo la reducción equimolar de los tioles libres por medio de una reacción de intercambio (Winther \& Thorpe, 2014). De 
estas interacciones químicas se obtiene como producto final de reacción el cromógeno acido 5-Tio-2-nitobenzoico (TNB), el cual posee un máximo de absorbancia a $412 \mathrm{~nm}$ (Rahman et al, 2007).

Se realizó una curva de calibración usando como referencia la glutationa, en un rango de concentración final de 0-100 nmol de glutationa para un volumen de $250 \mu \mathrm{L}$ en la celda correspondiente. De los extractos se adicionaron 100 $\mu \mathrm{L}$ de extracto a $1000 \mathrm{ppm}, 30 \mu \mathrm{L}$ de buffer y finalmente 125 $\mu \mathrm{L}$ de DTNB, se incubo la mezcla durante 15 minutos y se leyó la absorbancia a $412 \mathrm{~nm}$ en un espectrofotómetro de microplacas de 96 pozos (Multiskan Go, Thermo Scientific). Los análisis para cada extracto se realizaron por triplicado y en dos ensayos independientes.

\section{Cuantificación de Super Oxido Dismutasa (SOD)}

El sistema Xantina/Xantina oxidasa fue utilizado como fuente para la generación de un flujo de anión superoxido que oxida la hidroxilamina a nitrito. En consecuencia la concentración de nitrito puede ser medida debido al desarrollo de una coloración rosa, que varía su intensidad directamente con la concentración de nitritos, la cual puede ser medida mediante espectrometría UV/visible (Yang et al, 2004).

En esta determinación se realizó una curva de calibración usando SOD, en un rango de concentración de 0.0187-0.1497 Unit SOD . La mezcla se dejó reaccionar en lugar oscuro por $20 \mathrm{~min}$, transcurrido este tiempo se detuvo la reacción en un baño de hielo, en la celda correspondiente se adicionaron $100 \mu \mathrm{L}$ de ácido sulfanílico $19 \mathrm{mM}, 100 \mu \mathrm{L}$ de $\alpha-$ naftilamina $7 \mathrm{mM}$ y $100 \mu \mathrm{L}$ del estándar. En el caso del extracto, la muestra se preparó según lo descrito en la tabla 2, se realizó el mismo procedimiento que con los estándares, finalmente la mezcla contenida en las celdas se dejó reaccionar por $20 \mathrm{~min}$ y se leyó la absorbancia $529 \mathrm{~nm}$. Las muestras fueron evaluadas por triplicado y en dos (2) ensayos independientes.

Tabla 2. Preparación de la muestra para análisis SOD.

\begin{tabular}{cccccc}
\hline $\begin{array}{c}\text { Extracto } \\
\text { metanólico a } \\
\mathbf{1 0 0 0} \mathbf{p p m}(\mu \mathrm{L})\end{array}$ & $\begin{array}{c}\text { KCN en } \\
\text { Buffer de } \\
\text { fosfatos }(\mu \mathrm{L})\end{array}$ & $\begin{array}{c}\text { Agua } \\
(\mu \mathrm{L})\end{array}$ & $\begin{array}{c}\text { Xantina } \\
(\mu \mathrm{L}) \\
\mathbf{0 . 1 \%}\end{array}$ & $\begin{array}{c}\text { Cloruro de } \\
\text { hidroxilamina } \\
(\mu \mathrm{L}) \mathbf{1 ~ m M}\end{array}$ & $\begin{array}{c}\text { Xantina } \\
\text { oxidasa }(\mu \mathrm{L}) \\
\mathbf{4 . 1 6} \text { Units } / \mu \mathrm{L}\end{array}$ \\
\hline 5 & 150 & 40 & 15 & 15 & 75 \\
\hline
\end{tabular}

Evaluación de actividad antioxidante por el ensayo

Se preparó una solución de $\operatorname{DPPH}^{\bullet-}$ a $20 \mathrm{mg} / \mathrm{L}$ con metanol, justo antes de su utilización. En la celda correspondiente se adicionaron $25 \mu \mathrm{L}$ de extracto a $1000 \mathrm{ppm}$ y $100 \mu \mathrm{L}$ de solución de $\mathrm{DPPH}^{\bullet}$, la mezcla se incubo por 30 minutos en oscuridad y finalmente se leyó la absorbancia a $517 \mathrm{~nm}$ (Brand-Williams \& Berset, 1997). El control positivo utilizado fue hidroquinona a $1000 \mathrm{ppm}$, mientras que el control negativo usado fue metanol. Las muestras fueron evaluadas por triplicado y en dos (2) ensayos independientes.

\section{Determinación de fenoles totales}

Para la cuantificación del contenido fenólico se siguió la metodología descrita por (Magalhães et al, 2010), en la cual a $50 \mu \mathrm{L}$ del extracto a $100 \mathrm{mg} / \mathrm{L}$ se le adicionaron $50 \mu \mathrm{L}$ del reactivo de Folin-Ciocalteu $(1: 50, v / v)$ e inmediatamente después se adicionaron $100 \mu \mathrm{L}$ de una solución de $\mathrm{NaOH}$ $(0.35 \mathrm{M})$. Esta mezcla se dejó reaccionar por tres minutos y se midió la absorbancia a $760 \mathrm{~nm}$. Para esta determinación se realizó una curva de calibración de ácido gálico a 0, 2, 4, 8 y $16 \mu$ g Ácido gálico/mL. La determinación se realizó por triplicado y con dos repeticiones en tiempos diferentes.

\section{Análisis estadístico}

Se realizaron dos análisis independientes, cada uno con tres repeticiones por muestra, los datos fueron tratados con el software GraphPad Prism versión 5, se implementó el método de mínimos cuadrados para la búsqueda del modelo estadístico adecuado, mediante comparación de medias se corroboro la reproducibilidad de los métodos y finalmente se realizó la correlación entre las variables teniendo en cuenta el coeficiente de correlación de Pearson ( $R$ ), Todo el análisis estadístico se realizó para un $\alpha=0.05$.

\section{RESULTADOS Y DISCUSIÓN |}

Caracterización fitoquímica por cromatografía de capa delgada (CCD) de los extractos

En las muestras, se evidenciaron la presencia de los siguientes núcleos fitoquímico alcaloides, fenoles, flavonoides, saponinas y terpenos. Los fenoles, flavonoides y saponinas fueron los más abundantes como se muestra en la tabla 3.

\begin{tabular}{|c|c|c|c|c|c|c|c|}
\hline \multirow[b]{2}{*}{ Familia } & \multirow[b]{2}{*}{ Especie } & \multirow[b]{2}{*}{ \# UTP } & \multicolumn{5}{|c|}{ Núcleo fitoquímico ${ }^{a}$} \\
\hline & & & $\mathrm{A}$ & $F$ & $\mathrm{FI}$ & $\mathrm{S}$ & $\mathrm{T}$ \\
\hline \multirow[t]{2}{*}{ Euphorbiaceae } & $\begin{array}{l}\text { Hyeronnima } \\
\text { macrocarpa } \\
\text { Aspilia }\end{array}$ & 42 & + & + & + & + & + \\
\hline & $\begin{array}{l}\text { quinqueneruis } \\
\text { Mikania }\end{array}$ & 81 & - & + & - & - & - \\
\hline \multirow[t]{4}{*}{ Asteraceae } & $\begin{array}{l}\text { banisteriae } \\
\text { Baccharis }\end{array}$ & 124 & - & + & + & + & + \\
\hline & trinervis & 131 & - & + & - & - & - \\
\hline & $\begin{array}{l}\text { Tilesia baccata } \\
\text { Peperomia }\end{array}$ & 133 & - & - & - & + & + \\
\hline & $\begin{array}{l}\text { acuminata } \\
\text { Piper }\end{array}$ & 154 & + & + & + & + & + \\
\hline \multirow[t]{3}{*}{ Piperaceae } & $\begin{array}{l}\text { umbellatum } \\
\text { Piper }\end{array}$ & 163 & - & - & - & - & - \\
\hline & $\begin{array}{l}\text { crassinervium } \\
\text { Piper }\end{array}$ & 167 & - & + & + & + & + \\
\hline & calceolarium & 194 & - & - & + & + & + \\
\hline
\end{tabular}

Las saponinas son compuestos que las plantas producen como mecanismo de defensa contra patógenos y herbívoros (Augustin et al, 2011). Altos niveles de estos metabolitos, pueden estar influenciados por factores abióticos y bióticos como la temperatura, humedad, luz, fertilidad del suelo, competencia con cultivos o plantas vecinos e interacciones 
con organismos patógenos como bacterias, hongos, virus, nematodos e insectos (Szakiel et al, 2010).

\section{Cuantificación de tioles y SOD}

Los tioles de bajo peso molecular son de vital importancia en las células ya que regulan muchos procesos mediante reacciones redox (Poole, 2015). En las plantas se cuenta con la presencia de compuestos sulfurados que tienen funciones antioxidantes específicamente como es el caso de la cisteína y la glutationa (Venegas, 2012), teniendo en cuenta la importancia que representa este grupo de moléculas 32 especies fueron evaluadas por el método colorimétrico de Ellman's.

Los coeficientes de correlación de las curvas de calibración obtenidos para la cuantificación de tioles libres y de SOD fueron de 0.9992 y 0,9989 respectivamente, lo cual indica una buena linealidad (Ver Figura 1) y por ende que el modelo estadístico implementado es adecuado lo cual representa confiabilidad en las concentraciones calculadas para cada extracto de las plantas estudiadas.
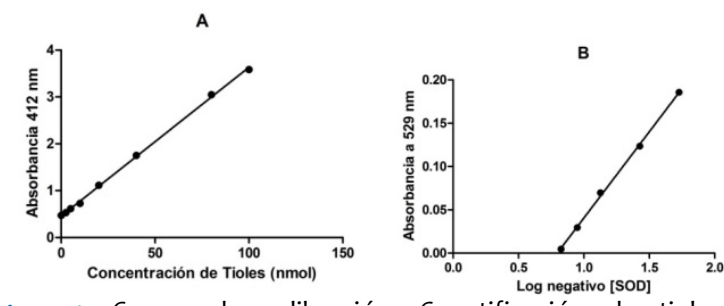

Figura 1. Curvas de calibración: Cuantificación de tioles (A) Cuantificación de SOD (B).

En las figuras 2 y 3 se presentan la concentración de los tioles libres y el contenido de moléculas con la capacidad de dismutar el radical superóxido en unidades de superóxido dismutasa (SOD) evaluada en las especies que presentaron una concentración de tioles mayor a $55 \mathrm{nmol} / \mathrm{mg}$ de extracto. Las especies de la familia Piperaceae fueron las que presentaron mayor contenido de tioles libres, seguidamente de las Asteraceae y Euphorbiaceae, respectivamente, como se puede observar en la figura 2.

Se destacan principalmente las especies Piperomia acuminata (UTP 154), Piper eriopodon (UTP 158), Piper umbellatum (UTP 163), las cuales presentan una concentración de tioles superior a $89 \mathrm{nmol} \mathrm{GSH} / \mathrm{mg}$ de extracto mientras que Piper Daniel-gonzalezii (UTP 197) presento la concentración de tioles más baja (20.2972 nmol $\mathrm{GSH} / \mathrm{mL}$ ). Respecto a las especies de la familia Asteraceae, las que presentaron alta concentración de tioles fueron Montanoa sp (UTP 80), Baccharis trinervis (UTP 131), Tilesia baccata (UTP 133) y Mikania llonensis (UTP 136) mayor a 90 $\mathrm{nmol} \mathrm{GSH/mg.} \mathrm{En} \mathrm{la} \mathrm{familia} \mathrm{Euphorbiaceae,} \mathrm{la} \mathrm{especie} \mathrm{Croton}$ magdalenensis (UTP 70) mostró la concentración de tioles más significativa de $101.8117 \mathrm{nmol} \mathrm{GSH} / \mathrm{mg}$ de extracto, como puede observarse en la figura 2. Teniendo en cuenta que se trabajó con los extractos metanólicos, estos resultados son razonables teniendo en cuenta que el grupo sulfihídrilo es análogo al grupo hidroxilo por ende poseen características químicas similares dentro de estas su polaridad y por esta razón se encuentran altas concentraciones de estas moléculas en este extracto.

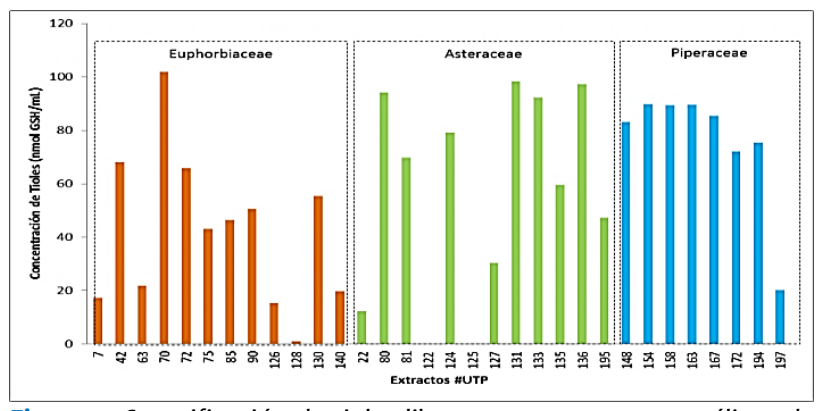

Figura 2. Cuantificación de tioles libres en extractos metanólicos de plantas de las familias Asteraceae, Euphorbiaceae y Piperaceae.

Entre tanto, la determinación de SOD se realizó en los extractos metanólicos de las especies vegetales que presentaron una concentración de tioles mayor a 55 $\mathrm{nmol} / \mathrm{mg}$ de extracto, lo cual corresponde a 17 especies, como se observa en la figura 3.

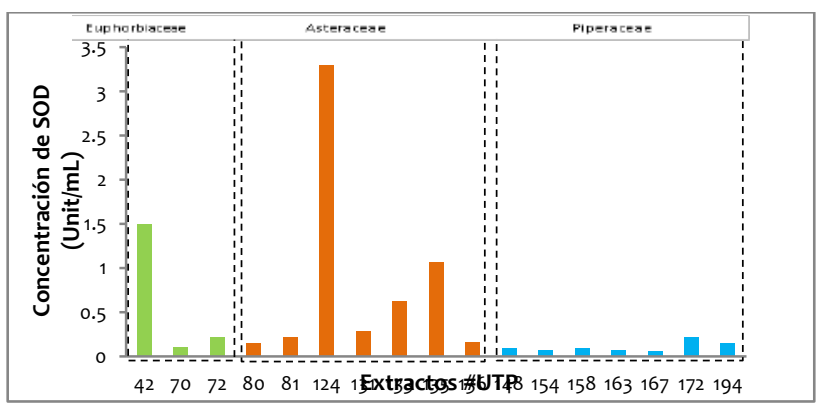

Figura 3. Cuantificación en unidades de SOD en extractos metanólicos de plantas de las familias Asteraceae, Euphorbiaceae y Piperaceae.

De los resultados obtenidos se destacan principalmente las especies Hyeronnima macrocarpa (UTP 42) de la familia Euphorbiaceae con una concentración de SOD de 1.4952 Units SOD/ mg de extracto, Mikania banisteriae (UTP 124) y Lepidaploa lehamannii (UTP 135) de la familia Asteraceae con una concentración dede 3,3045 y 1,0685 Units SOD/ mg de extracto, respectivamente.

Debido a que la funcionalidad de esta enzima está dada por su estructura cuaternaria, donde todos los cambios en el ambiente pueden generar un daño irreversible en mayor o menor media; modificando su estructura y en consecuencia su funcionalidad (Menvielle-Bourg, 2005). Cambios como la temperatura y $\mathrm{pH}$ a los cuales es sometido el material vegetal para la obtención del extracto, que se evidencian en la inactivación de la enzima, reflejada en las bajas concentraciones obtenidas para estos extractos estudiados, en el caso del extracto (UTP124) Mikania Bonistenae que presenta la concentración más alta, respecto a los demás extractos evaluados, se puede inferir que este contiene moléculas que actúan como miméticos de la SOD, es decir, 
que también poseen la capacidad de dismutar el radical superóxido.

\section{Evaluación de la actividad antioxidante}

En la figura 4 se presenta la capacidad antioxidante mediante el ensayo con DPPH de los extractos metanólicos que presentaron alto contenido de tioles libres.

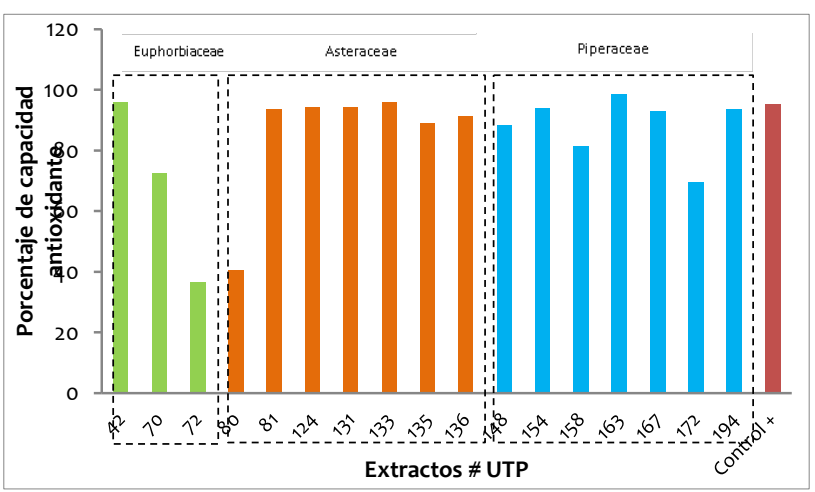

Figura 4. Capacidad antioxidante por DPPH de plantas con alto contenido de tioles libres.

Es de anotar que 9 especies presentan una buena capacidad antioxidante, exhibiendo un comportamiento similar a la hidroquinona empleada como control positivo (\%AA 95.3153).

Los extractos metanólicos de las plantas estudiadas exhiben un buen perfil de actividad antioxidante, con valores superiores al $90 \%$ mediante el ensayo con DPPH ${ }^{\circ}$. Valores que no pueden ser atribuidos únicamente a la acción de los compuestos tipo tiol y sistemas enzimáticos como la SOD, debido a que en los extractos crudos se tiene una mezcla de compuestos que pueden exhibir esta propiedad; como se puede observar en la tabla 3 con el contenido de fenoles totales.

Tabla 3. Contenido de tioles libres, fenoles totales y porcentaje de actividad antioxidante por el ensayo con DPPH.

\begin{tabular}{lccc}
\hline \multicolumn{4}{c}{ antioxidante por el ensayo con DPPH. } \\
\hline & $\begin{array}{c}\text { Tioles } \\
\text { (nmol GSH/ } \\
\text { mg extracto) }\end{array}$ & $\begin{array}{c}\text { Fenoles } \\
\text { ( } \begin{array}{c}\text { ag ácido gálico/ mg } \\
\text { extrato) }\end{array}\end{array}$ & \%AA \\
\hline Hyeronnima macrocarpa & 68,1033 & 21,215 & 95,9016 \\
Aspilia quinqueneruis & 69,7878 & 17,095 & 93,7106 \\
Mikania banisteriae & 79,1506 & 16,046 & 94,2623 \\
Baccharis trinervis & 98,4106 & 9,103 & 94,1019 \\
Tilesia baccata & 92,3361 & 14,239 & 95,9062 \\
Peperomia acuminata & 89,7539 & 20,080 & 93,9730 \\
Piper umbellatum & 89,5906 & 16,167 & 98,5895 \\
Piper crassinervium & 85,3789 & 19,441 & 92,9714 \\
Piper calceolarium & 75,4067 & 6,702 & 93,6134 \\
\hline
\end{tabular}

\section{CONCLUSIONES}

Las especies más destacadas por su contenido de tioles libres es Croton magdalenensis (UTP 70, Euphorbiaceae)
Para los extractos metanolicos de las especies Hyeronnima macrocarpa (UTP 42, Euphorbiaceae), Mikania banisteriae (UTP 124) y Lepidaploa lehamannii (UTP 135) de la familia Asteraceae se encontró la presencia de metabolitos con capacidad de neutalizar la acción del radical superóxido actuando de manera mimética a la enzima Superóxido dismutasa presente en los organismos vivos.

Con los datos obtenidos no es posible establecer una relación entre el contenido de los núcleos en estudio y la capacidad antioxidante de los extractos ya que se trabajó con los extractos crudos que están constituidos por gran cantidad de metabolitos secundarios que pueden contribuir a la actividad antioxidante de estos. Además, es de anotar que es el primer reporte de las especies estudiadas con estas propiedades; por lo cual se hace necesario realizar otras investigaciones que conduzcan a identificar los metabolitos secundarios con estas propiedades y de generar un potencial de uso de las plantas evaluadas. .

\section{AGRADECIMIENTOS}

A la Vicerrectoría de Investigaciones, Innovación y Extensión de la Universidad Tecnológica de Pereira y al proyecto: Desarrollo de capacidades científicas y tecnológicas en biotecnología aplicadas a los sectores de salud y la agroindustria en el departamento de Risaralda (Código: BPIN 201200010050) financiado por el Sistema General de Regalías. A las Corporaciones: Autónoma Regional de Risaralda (CARDER) y del Quindío (CRQ) por conceder el permiso para acceder a las plantas.

\section{CONFLICTO DE INTERESES}

Los autores no tienen conflicto de intereses.

\section{REFERENCIAS |}

- Augustin JM, Kuzina V, Andersen SB, Bak S. Molecular activities, 2011, Biosynthesis and evolution of triterpenoid saponins. Phytochemistry. 72(6):435-57.

- Brand-Williams , V., \& Berset , C. 1997. Kinetics and Mechanisms of antioxidant activity using DPPH • Free Radical Method. LWT - Science and Technology, 609-615.

- Gao , Y., Zhao , J., Zu, Y., Fu, Y., Liang , L., Luo , M, 2012, Antioxidant properties, superoxide dismutase and glutathione reductase activities in HepG2 cells with a fungal endophyte producing apigenin from pigeon pea [Cajanus cajan (L.) Millsp.], Food Reseach International, 49(1), 147-152

- Magalhães LM, Santos F, Segundo MA, Reis S, Lima JLFC. 2010, Rapid microplate high-throughput methodology for assessment of Folin-Ciocalteu reducing capacity, Talanta,83(2),441-7.

- Masi, A., Fabrega-Prants, M., \& Pivato, M, 2014, Low-Molecular-Weight Thiols In Plants: Fuctional And Analytical Implications. Archives Of Biochemistry And Biophisics, 83-99.

- Menvielle-Bourg, F, 2005, Superoxide Dismutase (SOD), a powerful antioxidant, is now available orally, Phytothérapie, 3, 1-4

- Niño, J., Mosquera, O., Correa, Y., \& Narváez, D. 2006, Antibacterial, antifungal and cytotoxic activities of eight Asteraceae and two Rubiaceae plants from Colombian biodiversity. Brazilian Journal of Microbiology, 37(4), 566-570. 
- Nordberg J., Arnér E.S.J. 2001. Reactive oxygen species, antioxidants, and the mammalian thioredoxin system. Free Radical Biology and Medicine. 31(11), 1287-1312.

- Poole, B. L, 2015, The basic of thiols and cysteines in redox biology and chemistry, Free Radical Biology and Medicine, 80, 148-157

- Rahman, I., Kode, A., and Biswas S. K, 2007, Assay for quantitative determination of glutathione and glutathione disulfide levels using enzymatic recycling method, Nature Protocols, 1, 3159-3165.

- Szakiel A, Pączkowski C, Henry M., 2010, Influence of environmental abiotic $f$ actors on the content of saponins in plants. Phytochemistry Reviews, 10(4), 471-91.

- Venegas, I, 2012, Efecto redox de diferentes extractos herbales sobre biomoléculas: Lípidos de membrana y tioles proteicos, Universidad de Chile, Departamento de Química Farmacológica y Toxicología.
- Wagner H, Bladt S, 1996, Plant Drug Analysis, B S Publishers

- Winther, J. R., \& Thorpe, C, 2014, Quantification of thiols and disulfides, Biochimica et Biophysica Acta, 1840(2), 838-846.

- Wu, J., Kosten, T. R., \& Zhang, X. Y., 2013, Free radicals, antioxidant defense systems, and schizophrenia, Progress in neuro-psychopharmacology \& biological psychiatry, 46(200), 200-206

- Yang, W.-M., Liu, J.-K., Qin, X.-D., Wu, W.-L., \& Chen, Z.-H. 2004, Antioxidant activities of three dihydrochalcone glucosides from leaves of Lithocarpus pachyphyllus. Zeitschrift für Naturforschung. C, Journal of biosciences, 481-4.

- Yen, G, 2000, Antioxidant activity of anthraquinones and anthrone. Food Chemistry, 70(4), 437- 441 